\title{
ARTICLE
}

\section{Perceptions of training in gonioscopy}

\author{
Rui Feng $\mathbb{1}^{1} \cdot$ Sheila M. H. Luk ${ }^{2} \cdot$ Clara Hoi Ka Wu $\mathbb{1}^{3} \cdot$ Laura Crawley $^{2} \cdot \operatorname{lan}$ Murdoch $\mathbb{E}^{4}$
}

Received: 22 June 2018 / Revised: 22 January 2019 / Accepted: 23 May 2019 / Published online: 2 July 2019

(c) The Author(s), under exclusive licence to The Royal College of Ophthalmologists 2019

\begin{abstract}
Purpose To evaluate ophthalmology trainees' perception of their gonioscopy learning experience in the Ophthalmology Specialty Training programme.

Materials and methods A cross-sectional electronic survey was conducted amongst ophthalmology trainees across London Deaneries. The ten questions survey collected parameters, including training grade, previous level of gonioscopy training, confidence in performing the procedure, level of satisfaction with the training formats received, potential barriers and improvements to the training programme. The respondents were also invited to express any additional comments.

Results Fifty-seven complete responses were analysed. The respondents included 25 junior trainees (ST1-3) and 32 senior trainees (ST4-7 and fellows). One-fifth of the respondents (11/57) were unconfident in performing gonioscopy, the majority being junior trainees $(9 / 11)$. Over a quarter of the respondents were dissatisfied with the quantity of the gonioscopy training received. Teaching formats, such as consultant teaching (mean $8.0 \pm 0.50$ ), self-directed learning (mean $8.0 \pm 0.38$ ) and small-group tutorials (mean 7.6 \pm 1.6 ) were all well received. Overall, lack of clinical time was considered as the major barrier to gonioscopy training; however, lack of training was considered as the major barrier in the low-confidence group. Conclusions This study highlighted ophthalmology trainees' dissatisfaction in the current gonioscopy training curriculum and a lack of confidence in performing the procedure. Appropriate modifications to the Ophthalmology Specialty Training programme could enhance trainees' gonioscopy learning experience.
\end{abstract}

\section{Introduction}

Glaucoma is one of the leading causes of irreversible blindness worldwide [1]. Studies have shown that diagnosis made based on symptoms alone often fails to correctly detect a common form of glaucoma - primary angle-closure glaucoma (PACG) [2, 3]. It is therefore vital for ophthalmologists to be experienced in the gold standard diagnosis tool, gonioscopy, as it is the only slit-lamp based method to

Supplementary information The online version of this article (https:// doi.org/10.1038/s41433-019-0498-8) contains supplementary material, which is available to authorised users.

Rui Feng

rui.feng1@nhs.net

1 Imperial College London, London, United Kingdom

2 Imperial College Healthcare NHS Trust, London, United Kingdom

3 City Hospitals Sunderland NHS Foundation Trust, Sunderland, United Kingdom

4 Moorfields Eye Hospital NHS Foundation Trust, London, United Kingdom allow direct examination of the anterior chamber angle, along with important information such as morphology, pigmentation and any other abnormality [4, 5]. NICE guidelines recommend all patients with a suspicious anterior chamber angle or previous inconclusive examinations to undergo gonioscopy at presentation [6]; however, recent research has indicated that its use in clinical practice has decreased [7-9]. The reasons postulated include the advancements in other modalities of angle assessments and patient refusal $[7,8]$. In this study, we aim to explore another dimension to this issue, namely the challenges faced by ophthalmology trainees in learning gonioscopy.

Recent ophthalmology training in the United Kingdom has undergone significant reformation since the introduction of Modernising Medical Careers (MMC), Ophthalmology Specialty Training (OST) Programme and European Working Time Directives (EWTD) [10]. Many believe these new agendas have significantly compromised the practical aspects of ophthalmology training [10-12]. The reduction of training hours is believed to have resulted in a fall of confidence amongst trainees in performing practical procedures including gonioscopy, a skill that requires regular practice and repeat reinforcement to master [13, 14]. 
Ophthalmologists are under enormous pressure to meet service provision targets [15], which further reduces valuable learning opportunities, and could potentially lead to anxiety in inexperienced trainees [13, 14].

Therefore, this study was designed to evaluate ophthalmology specialist trainees' perception of gonioscopy learning experience, including their performance and interpretation skills, with a view to identifying areas for potential improvement in the training programme.

\section{Materials and methods}

\section{Questionnaire}

An electronic questionnaire (Supplementary document 1) was distributed online via the survey tool Qualtrics in May 2015 to ophthalmology trainees across London Deaneries, using its internal mailing list. The completion of the questionnaire was anonymous, voluntary and non-incentivised.

Parameters collected include training grade, previous level of gonioscopy training and the level of satisfaction with the training received. Trainees self-evaluated their confidence levels in performing gonioscopy, and were subsequently asked for potential barriers and improvements in these areas. The response options for each question were on a Likert scale between 0 and 10 . The final section invited the respondent to express any additional opinions and comments regarding gonioscopy training.

\section{Statistical analysis}

Questionnaire results were collated using Qualtrics and extracted for further statistical analysis, using Stata software version 14.0 (www.stata.com). Respondents were divided according to their training grade into juniors (ST1-3) or seniors (ST4-7 and fellows). Basic analysis was performed for demographical data. Confidence and satisfaction was graded as 'poor' if rated 0-4, and reasonable if rated 5-10. Satisfaction levels between groups are expressed as mean \pm $2 \times$ standard error of mean. Chi-square tests were used for categorical data and unpaired $t$-test analyses were used for interval data. A $p$-value $<0.05$ was considered as statistically significant. The free text section was analysed thematically.

\section{Results}

One hundred questionnaires were distributed electronically to ophthalmology trainees in the North London and South London deaneries and 69 replies were received (69\%). Twelve participants commenced the questionnaire but only

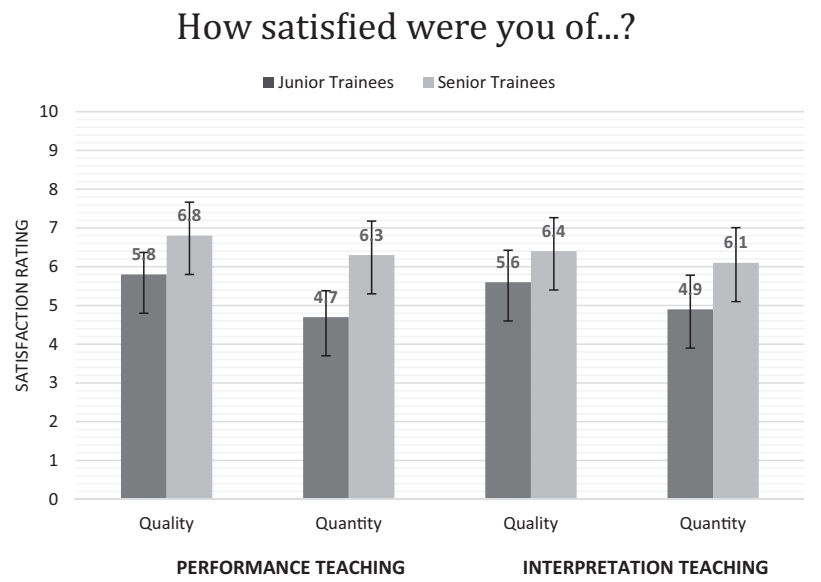

Fig. 1 Satisfaction levels of gonioscopy training in senior and junior trainees

responded to the first question, and were therefore excluded in the analysis. One reply was largely completed except the last two questions, and hence was included in the study. In total, 57 responses were included in the analysis (82.6\%). For the purposes of analysis, the respondents were divided into junior (ST1-3) trainees $(n=25)$ and senior (ST4-7 and fellows) trainees $(n=32)$.

Overall, $16(28.1 \%)$ and $17(29.8 \%)$ trainees were poorly satisfied (scores $<5)$ with the quantity of performance and interpretation training, respectively. Only $8(14.0 \%)$ and 11 $(19.3 \%)$ trainees were dissatisfied with the quality of performance and interpretation training. Satisfaction with gonioscopy training was variable amongst junior and senior trainees, as demonstrated by Fig. 1. Overall, junior trainees have significantly lower satisfaction than seniors in the quantity $(p=<0.01)$ and quality $(p=0.01)$ of performance training. For interpretation of gonioscopy, junior trainees also have lower satisfaction in the quantity and quality of teaching; however, this lower satisfaction is only statistically significant in the quantity $(p=0.04)$ and not in the quality of training $(p=0.07)$.

Figure 2 shows the distribution of respondents' training grades and their confidence in performing gonioscopy. Amongst junior trainees, two ST1s, five ST2 and two ST3 reported poor confidence $($ scores $<5)$ in performing gonioscopy (36\%). Only two trainees (one ST4 and one ST6) reported poor confidence (scores 5 or more) in the senior trainees group (6\%). All responses were divided into trainees with poor confidence in the technique and those with reasonable confidence. Those least confident were, unsurprisingly, significantly less satisfied in all aspects of their gonioscopy training, when compared with their counterparts (Fig. 3). In trainees with poor confidence, there was no statistical difference in their opinions between quantity and quality of performance and interpretation training $(p=0.55)$. 


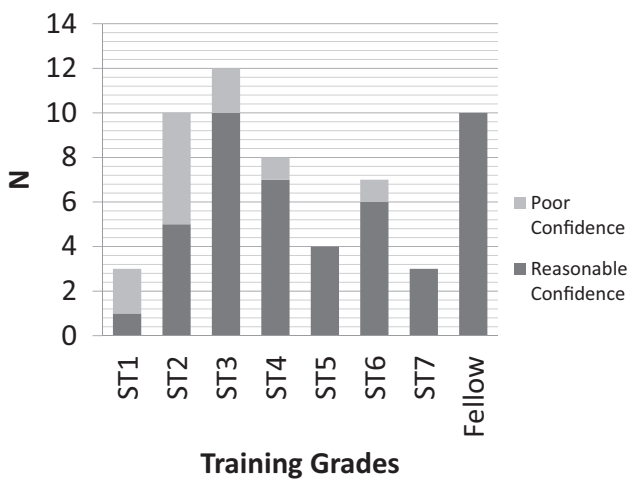

Fig. 2 Training grades of respondents and their confidence level in performing goniscopy

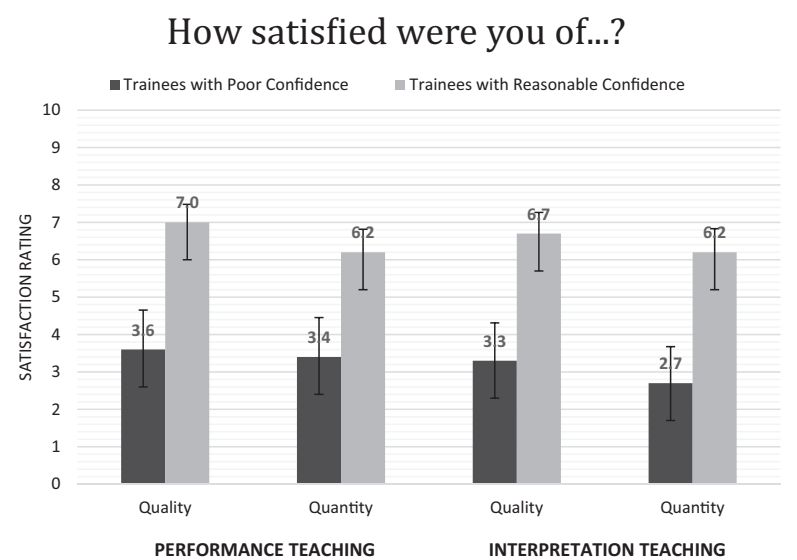

Fig. 3 Satisfaction levels of gonioscopy training in trainees with poor or adequate confidence in gonioscopy

Table 1 Methods of teaching received by trainees

\begin{tabular}{lccc}
\hline Teaching methods & \multicolumn{2}{l}{$\begin{array}{l}\text { Number of trainees who } \\
\text { received teaching }(\%)\end{array}$} & $X^{2} p$ value \\
\cline { 2 - 3 } & Junior $n=25$ & Senior $n=32$ & \\
\hline Consultant teaching & $18(72 \%)$ & $28(88 \%)$ & 0.14 \\
Self-directed learning & $22(88 \%)$ & $30(94 \%)$ & 0.45 \\
Small-group tutorial & $1(4 \%)$ & $9(28 \%)$ & $\mathbf{0 . 0 2}$ \\
Lectures & $17(68 \%)$ & $24(75 \%)$ & 0.56 \\
Books & $20(80 \%)$ & $28(88 \%)$ & 0.44 \\
Conferences & $3(12 \%)$ & $8(25 \%)$ & 0.22 \\
\hline
\end{tabular}

All trainees report on receiving a comparable amount of teaching in different formats, as demonstrated by Table 1 . The only significant difference between senior and junior trainees was in small-group tutorial teaching, where senior trainees $(28 \%)$ are more likely to receive this format of training than their junior $(1 \%)$ counterparts $(p=0.02)$. Trainees were subsequently asked to grade their satisfaction regarding the effectiveness of different teaching methods that they have received, on a scale 0-10 (Table 2).
Table 2 Satisfaction levels of teaching methods received by trainees

\begin{tabular}{llll}
\hline \multirow{2}{*}{ Teaching methods } & \multicolumn{3}{l}{ Satisfaction level $($ mean $\pm 2 \times$ SEM) } \\
\cline { 2 - 4 } & Total trainees & $\begin{array}{l}\text { Junior } \\
\text { trainees }\end{array}$ & $\begin{array}{l}\text { Senior } \\
\text { trainees }\end{array}$ \\
\hline $\begin{array}{l}\text { Consultant teaching } \\
\text { Self-directed }\end{array}$ & $8.0 \pm 0.50$ & $7.7 \pm 0.74$ & $8.3 \pm 0.66$ \\
learning & $8.0 \pm 0.38$ & $7.7 \pm 0.68$ & $8.4 \pm 0.42$ \\
Small-group & $7.6 \pm 1.6$ & 5.0 & $7.9 \pm 1.8$ \\
tutorials & & & \\
Lectures & $6.6 \pm 0.63$ & $6.0 \pm 0.91$ & $7.0 \pm 0.84$ \\
Books & $6.6 \pm 0.57$ & $6.7 \pm 0.74$ & $6.6 \pm 0.83$ \\
Conferences & $6.6 \pm 0.82$ & $7.0 \pm 1.2$ & $6.5 \pm 1.1$ \\
\hline
\end{tabular}

Comparing the mean satisfaction score of each option, trainees rated highly on consultant teaching $(8.0 \pm 0.50)$, self-directed learning $(8.0 \pm 0.38)$ and small-group tutorials (7.6 \pm 1.6 ), compared with lectures, books and conferences, all with means of 6.6 .

Respondents were asked to rate the extent to which each of the three barriers hindered their competence in performing gonioscopy. Lack of clinical time was the most highly rated barrier $(5.3 \pm 0.89)$, which followed difficulty of the task $(4.7 \pm 0.75)$ and lack of training hours $(4.4 \pm 0.77)$. Those with poor confidence recorded higher scores for each barrier $-p=0.09$ for lack of clinical time, $p=0.04$ for lack of training hours and $p=0.12$ for difficulty of the task.

Trainees were invited to rate the desired method to improve gonioscopy learning experience. All four suggested methods were rated favourably - the most popular was the 'use of patient images in camera during clinic with direct feedback' $(8.7 \pm 0.61)$. 'online materials' was the next most popular choice $(7.4 \pm 0.62)$ and 'small group tutorials' and 'surgical simulation' had an equal mean rating of 6.6 ( $2 \times$ SEM is 0.66 and 0.86 , respectively).

\section{Trainee's comment on their experience of gonioscopy training}

Thematic analysis of comments at the end of the questionnaire showed two main themes:

1. Junior trainee's dissatisfaction of learning gonioscopy 'As a ST2 I have found this a very difficult skill to become good at during busy glaucoma clinics, where I have been unable to get feedback on my findings.'

2. Suggestions of training methods to improve gonioscopy learning.

'Online videos were extremely useful, especially if the videos have arrows on them so you actually know what the voice over is referring too.' 
'Arrange a grand rounds session where gonioscopy can be performed on $8-10$ people with a range of angle grades and features, with a feedback session with images to follow.'

\section{Discussion}

This is the first study looking at ophthalmology trainees' perception and satisfaction of their gonioscopy learning experience in the United Kingdom. The results have highlighted significant shortcomings of gonioscopy training in the current OST curriculum. One-fifth of the respondents (11/57 trainees) were unconfident in performing gonioscopy, especially with junior trainees $(9 / 11$ trainees). This is an unsurprising finding as senior trainees are expected to have greater cumulative exposure and practice at performing gonioscopy than their junior counterparts, thus likely to be more confident. Importantly, over a quarter of the respondents were dissatisfied with the quantity of gonioscopy interpretation and performance teaching. Trainees with low confidence in performing gonioscopy are unsatisfied with both the limited training time and training quality in learning how to perform and interpret gonioscopy. A numerical target for performing gonioscopy may address the training time issue highlighted, which is a simple interim measure to implement [16]. Incorporating this into the OST curriculum would be worth considering, as it would be particularly beneficial for those with limited experiences and exposure to gonioscopy. More emphasis on gonioscopy training in the OST curriculum by the Royal College of Ophthalmology would ensure that more financial and human resources are introduced in teaching gonioscopy, which may consequently influence the quality of the gonioscopy learning experience for trainees, thus addressing training quality concerns raised by trainees with low confidence.

Teaching formats such as consultant teaching, selfdirected learning and small-group tutorials were all well received amongst trainees as effective methods of learning gonioscopy. Small-group tutorials were not rated quite as highly; however a much smaller number of mainly senior trainees had received this form of teaching. None in this study had yet received surgical simulation training for gonioscopy. When asked what method they felt would be beneficial, the trainees expressed a preference predominantly for consultant teaching, including live video images. This was also reviewed positively in the thematic analysis. Teaching equipment such as video camera, teaching scopes should be available in teaching hospitals to allow effective delivery of training to young ophthalmologists.
Surgical simulation, online materials and small-group tutorials were next in popularity. We would therefore recommend these teaching formats to be integrated into the OST curriculum for gonioscopy. More detail about online gonioscopy resource such as the www.gonioscopy.org should be provided by the curriculum to help trainees access these valuable materials early in the training. Surgical simulation is a rapidly evolving field in ophthalmology training and is gradually being adopted into the current curriculum, especially in phacoemulsification training $[17,18]$. Some have questioned its ability to replicated real clinical experience [19]; however, it could assist novices in developing a baseline level of skill $[17,18]$. This can then be transferred to clinical settings under supervision and could play a vital role in bridging the need for teaching with the limited resources [17, 20].

Most of the respondents rated the lack of clinical time as a major barrier to gonioscopy training. We suggest dedicated training clinics to provide sufficient time for trainees to acquire the skill of gonioscopy. Many junior trainees are now supernumerary to the service patient numbers in the clinic. We would hope that a resurvey would show that they feel less time pressured in glaucoma clinics, allowing trainees to practice gonioscopy under senior supervision in clinics as a result.

The method of assessment was not addressed in this study, but the use of a video slit-lamp or direct observational assessment may be the most appropriate method. This would formalise gonioscopy teaching and provide appropriate feedback, thus potentially improving satisfaction and confidence of the procedure in all trainees.

There are several limitations to this study. Firstly, due to the nature of the electronic survey, the reliance on selfreported data is subject to bias. Furthermore, this survey was only conducted in training deaneries within London and hence was limited in generalisation. Distributing this survey to trainees across the United Kingdom would allow the exploration of interregional training discrepancies. In addition, the respondents of this survey were still in their ophthalmology training. Our evidence suggests opinions concerning satisfaction and selfreported confidence in learning gonioscopy changes at different stages of training. Around a quarter of the questionnaires were not responded, and $10 \%$ of replies were excluded due to incomplete data. This may limit the ability of our study to reflect a comprehensive picture of the situation. However, we believe that using electronic questionnaires allows trainees to express their views anonymously and enhance our understanding of their true perceptions. Nevertheless, we feel the results of this study have provided valuable insights from trainees' perspective towards gonioscopy training under the current ophthalmology curriculum. 


\section{Conclusion}

In conclusion, this study has investigated specialist trainees' experience and views of training in gonioscopy. Junior trainees were less confident and more dissatisfied than senior trainees. We have identified areas of dissatisfaction within the current training and the preferred methods for acquiring the specific skill set needed for gonioscopy. Suggestions were made to both the OST curriculum and the clinical trainers to improve the current situation.

\section{Summary}

\section{What was known before}

- The use of gonioscopy in clinical practice has decreased, which may be due to the challenges faced by ophthalmology trainees.

\section{What this study adds}

- This is the first study focusing on ophthalmology trainees' perception and satisfaction of their gonioscopy learning experience in the United Kingdom. It identified the low-confidence level in performing and interpretation of gonioscopy, especially amongst junior trainees.

\section{Compliance with ethical standards}

Conflict of interest The authors declare that they have no conflict of interest.

Publisher's note: Springer Nature remains neutral with regard to jurisdictional claims in published maps and institutional affiliations.

\section{References}

1. World Health Organization. Global data on visual impairments 2010. Geneva: World Health Organization Organization; 2012.

2. Ong EL, Baasanhu J, Nolan W, Uranchimeg D, Lee P, Alsbirk $\mathrm{PH}$, et al. The utility of symptoms in identification of primary angle-closure in a high-risk population. Ophthalmology. 2008; 115:2024-9.

3. Friedman DS, Foster PJ, Aung T, He M. Angle closure and angle-closure glaucoma: what we are doing now and what we will be doing in the future. Clin Exp Ophthalmol. 2012;40: 381-7.

4. Friedman DS, He M. Anterior chamber angle assessment techniques. Surv Ophthalmol. 2008;53:250-73.

5. Spaeth GL. Gonioscopy: uses old and new. The inheritance of occludable angles. Ophthalmology. 1978;85:222-32.

6. National Institute for Health and Care Excellence. NG81 Glaucoma: diagnosis and management. U K: National Institute for Health and Care Excellence; 2017.

7. Coleman AL, Yu F, Evans SJ. Use of gonioscopy in medicare beneficiaries before glaucoma surgery. J Glaucoma. 2006;15: 486-93.

8. Foster PJ, Devereux JG, Alsbirk PH, Lee PS, Uranchimeg D, Machin D, et al. Detection of gonioscopically occludable angles and primary angle closure glaucoma by estimation of limbal chamber depth in Asians: modified grading scheme. Br J Ophthalmol. 2000;84:186-92.

9. Quigley HA, Friedman DS, Hahn SR. Evaluation of practice patterns for the care of open-angle glaucoma compared with claims data: the Glaucoma Adherence and Persistency Study. Ophthalmology. 2007;114:1599-606.

10. Rodrigues IA, Symes RJ, Turner S, Sinha A, Bowler G, Chan WH. Ophthalmic surgical training following modernising medical careers: regional variation in experience across the UK. BMJ Open. 2013; https://doi.org/10.1136/bmjopen-2013-002578.

11. Chand M, Faruque M, Dabbas N, Nash GF. Modernising medical careers and the British surgeons of the future. Br J Hosp Med. 2010;71:282-5.

12. Tsouroufli M, Payne H. Consultant medical trainers, modernising medical careers (MMC) and the European time directive (EWTD): tensions and challenges in a changing medical education context. BMC Med Educ. 2008;8:31-31.

13. Lagan J, Cutts L, Zaidi S, Benton I, Rylance J. Are we failing our trainees in providing opportunities to attain procedural confidence? Br J Hosp Med. 2015;76:105-8.

14. Turnbull A, Lash S. Confidence of ophthalmology specialist trainees in the management of posterior capsule rupture and vitreous loss. Eye. 2016;30:943-8.

15. Mehmood S, Anwar S, Ahmed J, Tayyab M, O'Regan D. A survey of UK surgical trainees and trainers; latest reforms well understood but perceived detrimental to surgical training. Surgeon. 2012;10:9-15.

16. Phillips AW, Madhavan A, Bookless LR, Macafee DA. Surgical trainers' experience and perspectives on workplace-based assessments. J Surg Educ. 2015;72:979-84.

17. Gibson A, Boulton M, Watson M, Moseley M, Murray P, Fielder A. The first cut is the deepest: basic surgical training in ophthalmology. Eye. 2005;19:1264-70.

18. Thomsen ASS, Bach-Holm D, Kjærbo H, Højgaard-Olsen K, Subhi Y, Saleh GM, et al. Operating room performance improves after proficiency-based virtual reality cataract surgery training. Ophthalmology. 2017;124:524-31.

19. Spitz L, Kiely E, Peirro A, Drake D, McAndrew F. Decline in surgical training. Lancet. 2002;359:83.

20. Schwab B, Hungness E, Barsness KA, McGaghie WC. The role of simulation in surgical education. J Laparoendosc Adv Surg Tech. 2017;27:450-4. 